\title{
Adaption of courses and learning environment to the user context in $\operatorname{dotLRN}$
}

\author{
David Huerva, Jeimy Vélez, Silvia Baldiris, Ramón Fabregat, David Mérida \\ Insitute of Informatics and Aplications (IIiA), Universitat de Girona \\ Edifici P-IV, Campus Montilivi, 17071 Girona, Spain \\ \{dhuervad;jeimy;baldiris;ramon;dmerida\}@eia.udg.edu
}

\begin{abstract}
The explosive growth of Internet during the last years has been reflected in the ever-increasing amount of the diversity and heterogeneity of user preferences, types and features of devices and access networks. Usually the heterogeneity in the context of the users which request web contents is not taken into account by the servers that deliver them implying that these contents will not always suit their needs. In the particular case of e-learning platforms this issue is especially critical due to the fact that it puts at stake the knowledge acquired by their users.

In the following paper we present a system that aims to provide the dotLRN e-learning platform with the capability to adapt to its users context. By integrating $\operatorname{dot} L R N$ with a multi-agent hypermedia system, online courses being undertaken by students as well as their learning environment are adapted in real time.
\end{abstract}

\section{Introduction}

During the last years Internet has experienced an explosive growth not only in the number of nodes that form part of it but also in terms of the diversity and heterogeneity of user preferences, type and features of devices and access networks.

The heterogeneity in the context of a user making a web request is usually not taken into account by the server that processes it, delivering exactly the same contents to all users. Thus in some cases a server will provide a content even when the user device is unable to display it appropriately due to limitations of the screen, storage or processing capabilities or because of the network state [1], [2]. This will cause the user to get confused or frustrated and often it will lead to a waste of resources. In the particular case of e-learning platforms, which use the Internet as the medium to publish their services, the problem caused by heterogeneity puts at stake the knowledge acquired by its users and is thus a critical issue that must be dealt with.

The system we present in this paper extends the capabilities of an e-learning platform known as dotLRN [3] to allow it to adapt in real time the learning environment and the learning resources it delivers to its users. In order to achieve this we have integrated it with a multi-agent adaptive hypermedia system known as MAS-SHAAD that has the capability of capturing contextual information of the users interacting with the dotLRN platform. The user context we have considered includes the technological features of the access device and the type and state of the access network on the user side and the load, type and state of the network on the server side.

\section{Description and architecture of the systems we are integrating}

dotLRN [3] is a LMS (Learning Management System) consisting in a portal system that integrates tools for the management of courses and contents as well as a set of collaborative tools. It works on OpenACS [4], [5], an open source framework designed to build web applications, implemented on AOLServer and a Potgres/Oracle relational database using the Tcl scripting language. The architecture of OpenACS (Figure 1) is composed of a set of individual packages amongst which are the system core, the content repository, the user and group management package, the request processor and the mechanisms to authenticate users and templates. dotLRN is also a OpenACS package which uses the services that other OpenACS packages provide. 


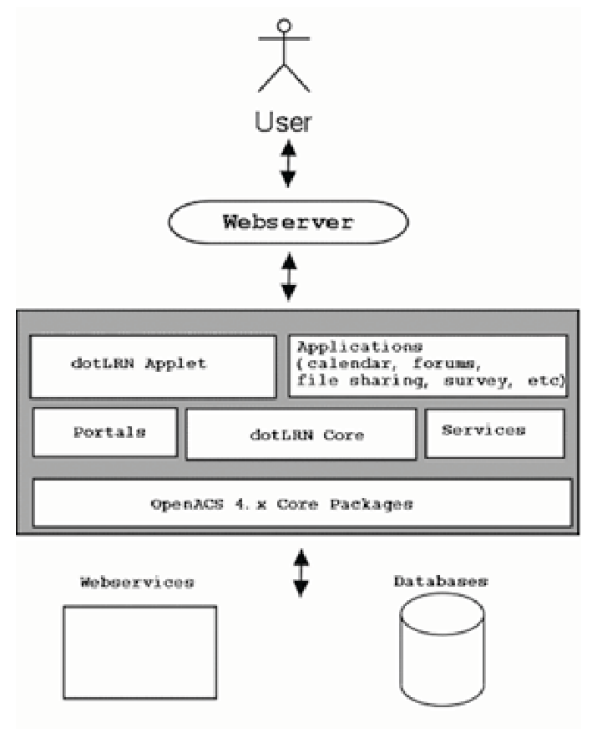

Figure 1. dotLRN / OpenACS architecture

Amongst the learning content specifications dotLRN can manage are IMS-LD (IMS - Learning Design) [6] and SCORM (Sharable Content Object Reference Model) [7] of which we have considered the former. IMS-LD is a specification done by the IMS Global Learning Consortium. It is an XML-based description for e-learning which provides a global framework for including the description of different pedagogical and methodological learning models. It specifies a set of learning activities, each of which is associated with a set of resources and services. It also establishes which roles can carry out these activities and under which conditions, setting a sequence of activities for every role.

The MAS-SHAAD system (Dinamical Adaptive Adaptable Hypermedia Multiagent System) [8], [9], [10] has been implemented with JADE [11] (Java Agent DEvelopment Framework) and performs content adaptation through the interaction of a set of intelligent agents running in the user's device and the webserver which stores the contents. Some of these agents have the task of gathering data about the context and then send it to an agent that infers how to dynamically build a HTML that suits best the user context.

\section{Integrating both systems together}

The communication between dotLRN and the MAS-SHAAD agent platform is carried out by means of the XML-RPC protocol [12] of which we use the Apache Java Implementation [13]. Through a simple set of data types and commands, XML-RPC allows a method to perform remote procedure calls using XML to codify them and HTTP as the transport mechanism. An OpenACS package has been implemented which registers a new filter in the OpenACS request processor pipeline. This filter is run for every request performed to the dotLRN platform and its goal is to capture data of the user request (user ID, URL of the user device profile and cookies). After the filter obtains the user request data it sends it to a servlet running in an Apache Tomcat server using the XML$\mathrm{RPC}$ protocol. When the servlet receives the request it checks if there is an agent in charge of handling it, if not it creates a new agent that will handle all the requests of the same user until its session expires. This agent then communicates with the user and server agents requesting them data about the context and forwarding it to a "decisor agent", which uses a set of conditional rules to infer the values of the adaption properties. These properties are then sent back to the OpenACS package through the Apache Tomcat servlet in order to use them in the adaptation process. Examples of properties we are considering in the current version of the system are the mime types of the resources, their resolution and their size.

In order to describe the features of the user's access device, UAProf [14] (User Agent Profile) profiles are used. They are based in CC/PP [15] (Composite Capability Preference Profiles) and provide information about the software, hardware and networking capabilities of the device. As we introduced earlier on, when a dotLRN user performs a request, the URL of its device profile is recovered from the headers of the request and is forwarded to its associated agent in the MAS-SHAAD system. This agent checks if the profile is available in the device repository, if not it downloads it from the given URL and stores it. Given the case that the device profile can't be recovered, the agents on the user's side are queried for data about the user's device. In Figure 2 we show an overview of the system and the communication between its main components.

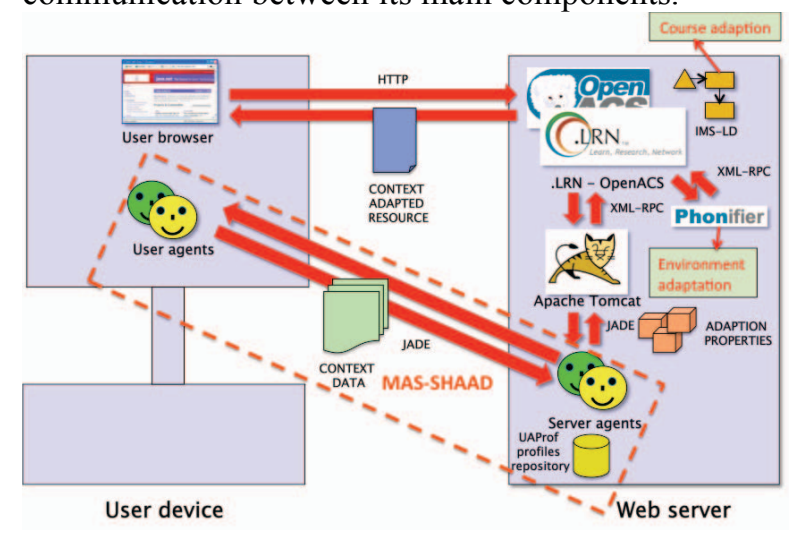

Figure 2. The system presented 


\section{The content adaptation}

Once the adaptation properties generated by the agent system have been sent to the OpenACS package, the adaptation process is carried out in two phases: learning environment adaptation and course adaption.

\subsection{Learning environment adaptation}

In this phase the general layout of the learning environment is adapted. This is done using the following two mechanisms: the OpenACS template system and a HTML transcoder.

The goal of OpenACS' template system is to split application logic from presentation logic. The template system allows that in OpenACS sites all code related to database manipulation or the state of the application is stored in a tcl file while the code to display the state of the application, basically HTML with a set of custom OpenACS tags, is stored in a ADP template. In order to give a uniform look to all pages of a website, OpenACS uses a mechanism of templates inclusion which allows to gather the common contents of the pages of a site, as for instance headers, footers and menus in "Master templates" which are then reused throughout all the pages of a site. The idea is that the common elements are grouped inside the "Master template" then every page of the site takes this master template and fills it with its specific contents. By adapting the set of master templates of a site we can adapt its global presentation without having to adapt every specific component. In our system we do this by conditioning how the components of the template are rendered to the value of the adaptation properties sent by the MAS-SHAAD system.

If the user is using a handheld device to interact with the system we also use an HTML transcoder known as Phonifier [16] to recodify the HTML pages generated by the systen before they are delivered to him. The goal is that that they render more suitably to its device. In our system we use a customized version of Phonifier which we have parametrized in order that it takes into acount the adaptation properties generated by the decisor agent.

\subsection{Course adaptation}

In the second phase the IMS-LD of a particular course being undertaken by a user is provided with the value of the adaptation properties. The possibility of defining personal global properties in an IMS-LD allows to create dynamic IMS-LDs which change the sequence and the contents presented to the user depending on the value these properties take. For instance we have considered a "screen resolution" property that IMS-LDs evaluate in order to choose a resource from a set of equivalent resources, all of which explain the same concept but that are suitable for different resolutions.

\section{Results}

Despite that the development of the system we presented has not yet ended we already managed to obtain some results. The version of the system we currently have implemented allows the adaption in real time of an IMS-LD as well as the user's learning environment. In order to do so it uses the adaptation properties generated by the MAS-SHAAD by using the information in the user's device profile.

We run some tests using a custom IMS-LD in which we created a set of conditions that hide or show the course contents depending on the values of the of the adaptation properties provided by the MASSHAAD system.

\section{Future work}

Although the core capabilities of the system have been implemented there is still much work to be done. The agents which recover data of the type and state of the network, on both the user and server side, have to be implemented as well as the agent which monitors the server load. Also we intend to implement a module that allows to automatically generate dynamical IMS-LDs from the characterized contents stored in a content repository known as Fedora Commons [17].

\section{References}

[1] Brusilovsky, P. and Maybury, M.: "The Adaptive Web", Communications of the ACM 45, 5. (2002)

[2] Oppermann, R.; Rashev, R. and Kinshuk: "Adaptability and Adaptativity in Learning Systems". Knowledge Transfer (Volume II) (Ed. A. Behrooz) pAce London UK, 1997, pp. 173-179

[3] Santos, O.C., Boticario, J.G., Raffenne, E., Pastor, R. Why using dotLRN? UNED use cases. FLOSS International Conference, 2007.

[4] OpenACS, http://openacs.org/

[5] Calvo, R.; Ghiglione, E. and Ellis, R.: "The OpenACS e-learning infrastructure" AusWeb 2003. The Ninth Australian World Wide Web Conference (2003) 
[6] IMS Learning Design. Version 1.0 Final Specification, 2003. http:/www.imsglobal.org/learningdesign/

[7] SCORM 2004, Sharable content Object Reference Model, Impact Summary. Version 1.0.

http://www.ADLNet.gov/scorm

[8] Mérida, D.; Huerva, D.; Fabregat, R.; Vélez, J. and Jové, T: "Adaptación de contenidos web considerando las características de los dispositivos de acceso". Published at Quinta Edición Revista Enlace Informático - Universidad del Cauca. ISSN. 1692-374X, 2006

[9] Mérida, D.; Fabregat, R. and Marzo, J.L.: "SHAAD: Adaptable, Adaptive and Dynamic Hypermedia System for content delivery". Workshop on Adaptive Systems for Web Based Education. WASWE2002. Málaga España, 2002

[10] Mérida, D.; Cannataro, M.; Fabregat, R. and Arteaga, C.: "MAS-SHAAD a Multi-agent System Proposal for an Adaptive Hypermedia System". Proceedings of IJCEELL journal Special issue: Adaptivity in Web and Mobile Learning Services, 2004
[11] Java Agent DEvelopment Framework (JADE): an Open Source platform for peer-to-peer agent based applications. JADE web site http://jade.tilab.com/

[12] Dave Winer. XML-RPC specification. 1999. (http://www.xmlrpc.com/spec)

[13] Apache XML-RPC version 3. http://ws.apache.org/xmlrpc/index.html

[14] User Agent Profiling Specification (UAProf). Version 20-October-2001.

http://www.openmobilealliance.org/tech/affiliates/wap/wap248-uaprof-20011020-a.pdf

[15] CC/PP Information Page. (http://www.w3.org/Mobile/CCPP/)

[16] Phonifier, http://phonifier.com/

[17] Fedora Commons, http://www.fedora.info/ 